\title{
Polariton superregular breathers in a resonant erbium-doped fiber
}

\author{
Yang Ren, ${ }^{1,2}$ Chong Liu, ${ }^{1,2,3,{ }^{*}}$ Zhan-Ying Yang,,${ }^{1,2, \dagger}$ and Wen-Li Yang ${ }^{1,2,4}$ \\ ${ }^{1}$ School of Physics, Northwest University, Xi'an 710069, China \\ ${ }^{2}$ Shaanxi Key Laboratory for Theoretical Physics Frontiers, Xi'an 710069, China \\ ${ }^{3}$ Optical Sciences Group, Research School of Physics and Engineering, The Australian National University, \\ Canberra, ACT 2600, Australia \\ ${ }^{4}$ Institute of Modern Physics, Northwest University, Xi'an 710069, China
}

(Received 23 June 2018; published 28 December 2018)

\begin{abstract}
We study polariton superregular (SR) breathers triggered by the resonant interaction of an optical field with two-level doping ions in an erbium-doped fiber. We demonstrate explicitly that the optical wave component always features a bright structure that describes the modulation instability (MI) development from localized small perturbations, while the SR matter-wave breathers exhibit more complicated dynamical evolution, which are absent in standard scalar SR breather theory. Despite the complexity, the exact link between these SR breathers and MI is established strictly by showing that the absolute difference of group velocities of SR breathers coincides exactly with the linear MI growth rate. In particular, we show how a small dip perturbation of the dark SR breather in the matter wave component induces an intriguing MI process of completely exponential decay. These results will enrich our understanding on the MI development in complex coupled light-matter interaction systems.
\end{abstract}

DOI: 10.1103/PhysRevE.98.062223

\section{INTRODUCTION}

Modulation instability (MI), i.e., the instability of a constant background with respect to infinitesimal periodic or irregular noisy perturbations, is a central process in nonlinear physics that has been observed in many different physical settings, including hydromechanics, optics, plasma, and BoseEinstein condensates [1,2]. This dynamics evolution can be modeled by the standard scalar nonlinear Schrodinger (NLS) equation [2-8]. In general, the property of initial linear MI stage (i.e., the MI criterion and growth rate) can be studied readily by the linear stability analysis. However, exact descriptions of the complete MI process involving both linear and nonlinear stages are long-standing challenges and are far less common [9].

One class of these unusual exact MI descriptions is the so-called breather [10-16], i.e., nonlinear oscillating structures on a plane-wave background of the standard NLS equation (confusion should be avoided between these waves and "discrete breather state" in lattices $[17,18]$ ). It should be emphasized that not all breathers, however, can describe the complete MI process - only a few unique ones, including the well-known Akhmediev breather [11,13], Peregrine breather [19], their higher-order counterparts [20-22], as well as the newly proposed superregular (SR) breather [14-16]. Note that these breathers are exact solutions of discrete spectrums. Another type of MI descriptions is the continuous spectrum waves obtained by asymptotic analysis of a class of localized perturbations [23].

\footnotetext{
*chongliu@nwu.edu.cn

†zyyang@nwu.edu.cn
}

For the exact MI description of breathers, the Akhmediev breather describes an elementary MI with one growth-return cycle evolved from a weak periodic perturbation [11,13]. Its limiting case with infinite period-the Peregrine breather-is therefore the simplest rigorous description of a MI process from localized single-peak weak perturbations, which corresponds to a particular MI band, i.e., the baseband (zerofrequency) MI regime [24]. Correspondingly, their higherorder modes reveal the nature of higher-order MI [20] and rogue wave events [25], respectively. However, these two MI scenarios have their own limitations. Namely, the Akhmedievtype breathers require an initial periodic perturbation in the whole infinite space; while the Peregrine-type breathers merely describe the particular zero-frequency MI. Nevertheless, this restriction can be overcome when one considers a MI scenario of so-called SR breathers $[14,15]$. The latter describes MI evolution from a localized small-amplitude perturbation that can cover the whole MI band. Indeed, the dynamical observation of SR breathers has be confirmed in both optics and hydrodynamics based on the standard scalar NLSE [16]. The MI nature has been shown strictly by establishing the exact link between MI growth rate and the absolute difference of group velocities of SR breathers [26]. Even for a noise-driven initial state, SR breathers can been excited and describe the edge state of nonlinear stage [27]. Moreover, the utility of SR breathers in generating rogue wave events [28] and the integrable extensions of SR breathers with higher-order effects $[26,29,30]$ have been revealed.

However, to model more general and complex classes of physical systems, investigation of MI in vector coupled systems is both relevant and necessary. In contrast to the scalar NLS case, coupled systems possess some additional coupling parameters and allow for resonant interaction 
between different components, which potentially yield rich and significant MI dynamics [31-42]. Indeed, even for the initial linear stage obtained by the simple linear stability analysis, the MI properties (criterion and growth rate) are changed greatly by the weak coupled-NLS interaction $[38,39]$ or the strong three-wave resonant [40]. However, recent studies demonstrate that the analog of Peregrine breathers in coupled complex systems exhibits structural diversity beyond the reach of the scalar NLS model, including the dark and four-petaled structures, and their coexistence $[39,41,42]$. Therefore, it is expected that SR breathers in these cases can exhibit nontrivial dynamics.

The light-matter interaction between optical fields with nonlinear resonant media is a central topic of research in physics. In general, we have complex from electromagnetic field and resonant medium polarization. These objects are spoken of as "polaritons" $[43,44]$. One of the most typical examples is the resonant interaction of pulses with twolevel atoms or ions governed by the Maxwell-Bloch (MB) system [45-48]. An otherwise absorbing material in this system becomes completely transparent for optical pulses of duration shorter than all the relevant relaxation times of the medium. This is now known as self-induced transparency (SIT) [49]. The resulting localized mode is the so-called SIT sech-shaped soliton [50]. The soliton control can be enhanced by multiple atom doping, if one extends the MB system into its multicomponent counterpart [51]. It has recently been demonstrated to admit breather solutions [33,52-55]. However, SR polariton breathers have not been studied.

In this paper, we study SR breathers formed by the resonant interaction of an optical field with two-level doping ions in an erbium-doped fiber generalizes the above MB case by including the group-velocity dispersion (GVD) and the Kerr nonlinearity. We demonstrate explicitly that the optical wave component always features a bright structure that describes the MI development from localized small perturbations, while the SR matter-wave breathers exhibit more complicated dynamical evolution. SR breathers in each component involve the half-transition and bound states arising from the resonant interaction. Despite the complexity, the exact link between these SR breathers and MI is established strictly by showing that the absolute difference of group velocities coincides exactly with the linear MI growth rate. Further, we show how a small dip perturbation of dark SR breathers in the matter wave component induces an intriguing MI process of completely exponential decay of perturbation.

\section{THEORETICAL MODEL}

The propagation of optical field in a nonlinear dispersive medium doped with two-level atoms (or ions) will be described by a non-integrable MB or NLS-MB equation. However, due to optical pulses of duration shorter than the dipole and population decay times, it provides a solvable model for the investigation of SIT. Further making the slowly varyingenvelope and rotating-wave approximations, the optical field propagation in an ion-doped medium is governed by the following coupled NLS-MB equations [45,46,56,57]:

$$
\begin{aligned}
& i U_{z_{0}}-\frac{\beta_{2}}{2} U_{t_{0} t_{0}}+\gamma|U|^{2} U+\frac{\hbar F \Omega_{0}^{2} n}{2 c p_{21}}\left\langle V_{1} V_{2}^{*}\right\rangle=0, \\
& V_{1 t_{0}}=\frac{i}{2} \delta V_{1}+\frac{2 i p_{21}}{\hbar F} U V_{2}, \\
& V_{2 t_{0}}=-\frac{i}{2} \delta V_{2}+\frac{2 i p_{21}^{*}}{\hbar F} U^{*} V_{1},
\end{aligned}
$$

where $U$ is the complex envelope with $z_{0}$ being the propagation distance and $t_{0}$ being the retarded time. $V_{1}$ and $V_{2}$ are the wave functions for the lower and upper levels, respectively. One requirement for the wave functions is that the total probability of finding a dopant atom in either the upper or lower levels is equal to unity, namely, $\left|V_{1}\right|^{2}+\left|V_{2}\right|^{2}=1$. The asterisks $*$ denote complex conjugation and brackets \langle\rangle is a local average over two-level systems. Parameters $\delta, \hbar, c$, and $n$ are constants that are responsible for the laser detuning to resonance, Planck constant divided by $2 \pi$, speed of light in vacuum, and linear refractive index of medium, respectively. $p_{21}=p_{12}$ is the complex dipole matrix element of transition between upper and lower states. Parameters $\beta_{2}, \gamma, \Omega_{0}, F$ are constants which are account for the GVD, Kerr nonlinearity, characteristic frequency, and scaling factor, respectively.

Note that $\beta_{2}>0\left(\beta_{2}<0\right)$, corresponds to the cases of anomalous (normal) dispersion. As in most NLS frameworks, we confine our discussion to the anomalous dispersion. By virtue of the following transformations of variables [41],

$$
\begin{aligned}
& E=\frac{U}{\sqrt{p_{s}}}, \quad P=\frac{i\left|p_{21}\right|}{p_{21} \sqrt{\sigma}} V_{1} V_{2}^{*}, \\
& 2 \eta=\left|V_{1}\right|^{2}-\left|V_{2}\right|^{2}, \quad z=\frac{z_{0}}{z_{s}}, t=\frac{t_{0}}{t_{s}},
\end{aligned}
$$

the coupled Eqs. (1) can be normalized to the dimensionless form

$$
\begin{aligned}
E_{z} & =i\left(\frac{\sigma}{2} E_{t t}+\frac{p_{s}}{p_{n}}|E|^{2} E\right)+2\langle P\rangle, \\
P_{t} & =2 i \omega P+\frac{2}{\sigma} E \eta, \\
\eta_{t} & =-\left(E P^{*}+P E^{*}\right),
\end{aligned}
$$

where $t_{s}$ is a typical pulse duration, which defines the unit time scale, $\omega=\delta t_{0} / 2$ is the scaled laser detuning. $z$ and $t$ represent the propagation distance and retarded time, respectively. $p_{n}=$ $\frac{1}{z_{s} \gamma}$ is the peak power of the usual NLS soliton, while $p_{s}=$

$\frac{\hbar^{2} F^{2}}{4 \sigma \tau_{s}^{2}\left|p_{21}\right|^{2}}$ denotes the peak power of the SIT soliton when the NLS component is absent. Other parameters,

$$
z_{s}=\frac{2 c}{\sigma n t_{s} \Omega_{0}^{2}}, \quad z_{d}=\frac{t_{s}^{2}}{\left|\beta_{2}\right|}, \quad \sigma=\frac{z_{s}}{z_{d}},
$$

are the SIT length, the dispersion length, and the scaled dispersion, respectively. Furthermore, according to the relationship between the wave functions, $P$ (which is related to the off-diagonal element) and $\eta$ (which signifies the local population difference between the upper and lower levels) must satisfy the condition of probability conservation [45]. It can be rewritten as

$$
4 \eta^{2}+4 \sigma|P|^{2}=1 .
$$


For the sake of simplicity, we assume $\langle P\rangle=P$ when the Lorentzian line profile (homogeneous broadening) or Gaussian line profile (inhomogeneous broadening) has an infinitesimal scale parameter. However, the peak power of the SIT soliton should be equal to that of the NLS soliton for a given input pulse width $[56,58]$. It is now evident that as the condition

$$
p_{n}=p_{s} \text {, i.e., } \gamma=\frac{4\left|p_{21}\right|^{2}\left|\beta_{2}\right|}{\hbar^{2} F^{2}},
$$

is met, Eq. (3) can be reduced to the integrable form

$$
\begin{aligned}
E_{z} & =i\left(\frac{1}{2} E_{t t}+|E|^{2} E\right)+2 P, \\
P_{t} & =2 i \omega P+\frac{2}{\sigma} E \eta, \\
\eta_{t} & =-\left(E P^{*}+P E^{*}\right) .
\end{aligned}
$$

Here we consider $\sigma=1$ without losing generality. In the following, we shall first shed light on the properties of the fundamental breathers in the coupled NLS-MB systems, since SR breathers are formed by a particular nonlinear superposition of fundamental breathers.

\section{FUNDAMENTAL BREATHERS AND THEIR CLASSIFICATION}

The fundamental breather solutions are constructed on the plane-wave backgrounds,

$$
E_{0}=a e^{i \theta}, \quad P_{0}=i k E_{0}, \quad \eta_{0}=\omega k-q k / 2,
$$

where $\theta=q t+v z, v=a^{2}+2 k-q^{2} / 2$. Here $a$ and $q$ denote the amplitude and frequency of the electric field $E_{0}, k$ is a real parameter which is related to the background amplitude of $P_{0}$ and $\eta_{0}$ components. Since $P$ and $\eta$ are balance in spatiotemporal amplitude distributions, i.e., $4 \eta^{2}+4|P|^{2}=1$, we fix $k=1 / \sqrt{(q-2 \omega)^{2}+4 a^{2}}$, without losing physics. This parameter relation will hold for all analysis in what follows.

The analytical fundamental breather solutions $E_{1}, P_{1}$, and $\eta_{1}$ on the backgrounds Eq. (8) are obtained by the standard Darboux transformation [see Appendix A], but the spectral parameter $\lambda$ parameterized by the Jukowsky transform $[14,15]$,

$$
\lambda=-\frac{i a}{2}\left(\Theta+\frac{1}{\Theta}\right)-\frac{q}{2}, \Theta=\operatorname{Re}^{i \alpha},
$$

where $R \geqslant 1, \alpha \in(-\pi / 2, \pi / 2)$ are the radius and angle of the polar coordinates.

Thus, this solution depends on $a, q, \alpha, R, \omega$, and the internal phase $\theta_{1}$. To analyze the property of fundamental breathers, we first let $\theta_{1}=0$, which results in that the central position of breathers is located at $(z, t)=(0,0)$. Then the structure property of breathers can be achieved by the Hessian matrix $[59,60]$. In principle, we need do this for all NLS-MB components. However, since the optical wave component $E$ always features a bright structure and the matter wave components $P$ and $\eta$ are spatiotemporally balanced in amplitude distributions, we prefer the matter wave component $\eta$, without loss of generality. The details are as follows.
The center point $\eta_{1}\left(\sigma_{1}, \sigma_{2}\right)=(0,0)$ is the critical point, and its value is given by

$$
\eta_{1}(0,0)=\frac{1}{2} k \frac{4 a \rho \Delta-\varpi\left(\Delta^{2}-\rho^{2}\right)}{\Delta^{2}+\rho^{2}},
$$

where $\Delta=\varrho-q+2 \omega, \varpi=q-2 \omega$. The structure property of the breather $\eta_{1}$ is determined by the following Hessian matrix:

$$
H_{\eta}=\left[\begin{array}{ll}
\left(\eta_{1}\right)_{\sigma_{1} \sigma_{1}} & \left(\eta_{1}\right)_{\sigma_{1} \sigma_{2}} \\
\left(\eta_{1}\right)_{\sigma_{2} \sigma_{1}} & \left(\eta_{1}\right)_{\sigma_{2} \sigma_{2}}
\end{array}\right]
$$

where

$$
\begin{aligned}
& \left(\eta_{1}\right)_{\sigma_{1} \sigma_{2}}=\left(\eta_{1}\right)_{\sigma_{2} \sigma_{1}}=\frac{2 k \rho^{2}}{\left(\rho^{2}+\Delta^{2}\right) r^{2}}\left(\Lambda_{1}-\delta \varpi\right), \\
& \left(\eta_{1}\right)_{\sigma_{1} \sigma_{1}}=\frac{-2 k \rho}{\left(\rho^{2}+\Delta^{2}\right) r^{2}}\left\{2 a \rho \delta+\Delta \Lambda_{2}+\left(R^{2}-1\right)^{2} \rho \varpi\right\}, \\
& \left(\eta_{1}\right)_{\sigma_{2} \sigma_{2}}=\frac{2 k \rho}{\left(\rho^{2}+\Delta^{2}\right) r^{2}}\left\{2 a \rho \delta-\Delta \Lambda_{2}-4 R^{2} \sin ^{2} \alpha \rho \varpi\right\},
\end{aligned}
$$

where $\quad r=1+R^{2}-2 R \cos \alpha, \Lambda_{1}=a\left[\left(R^{2}-1\right)^{2}-4 R^{2}\right.$ $\left.\sin ^{2} \alpha\right], \Lambda_{2}=a\left[\left(R^{2}-1\right)^{2}+4 R^{2} \sin ^{2} \alpha\right], \delta=2 R\left(R^{2}-1\right)$ $\sin \alpha$. Then, the structure criterion based on the Hessian matrix can be written explicitly as follows. If $H_{\eta}$ is a negative definite matrix, i.e., the eigenvalues are both negative, $\eta_{1}(0,0)$ is the local maximum, thus the breather is the standard "bright" one with a hump and two valleys [11]. If $H_{\eta}$ is a positive definite matrix, i.e., the eigenvalues are both positive, $\eta_{1}(0,0)$ is the local minimum, the breather is the so-called "dark" one [34]. If $H_{\eta}$ is an indefinite matrix, i.e., the two eigenvalues have different signs, $\eta_{1}(0,0)$ is a saddle point, the breather is the "four-petaled" one [39]. The amplitude characteristic of fundamental breathers in each component versus the background frequency $q$ is shown in Fig. 1.

As is shown clearly in Fig. 1, optical wave component $E$ always features a bright structure (top row), while the matter wave components $P$ and $\eta$ exhibit rich dynamics (middle and bottom rows). Specifically, the component $\eta$ shows a four-petaled structure [see Fig. 1(d)] in the range $q \in$ $\left(q_{1}, q_{2}\right)$, where $q_{1}=X_{1}-X_{2}, q_{2}=X_{1}+X_{2}, X_{1}=2 \omega+$ $\rho(x-a) /(2 x), X_{2}=\rho\left(\varrho^{2}-4 a x\right)^{\frac{1}{2}} /(2 x)$, and $x=\rho-a$. However, as $q>q_{2}\left(q<q_{1}\right)$, it exhibits a bright (dark) structure [see Figs. 1(e) and 1(c)]. Due to the complementary relation between $P$ and $\eta$, i.e., $4 \eta^{2}+4|P|^{2}=1$, one can then readily obtain the complementary breather structures for the component $P$. It should be pointed out that, although both $P$ and $\eta$ are four-petaled structures, the topology is different. Namely, the $\eta$ four-petaled breather is formed by two humps and two valleys, while the $P$ one is composed of four valleys [see Fig. 1(d)]. This can be proved easily by the complementary relation.

Note also that the fundamental breather can convert to the nonbreathing mode when $V_{g r 1}=V_{p h 1}$ (thus $q=q_{s}$, where $q_{s}$ satisfies the equation $\left.\left[\rho^{2}+(\varrho-\varpi)^{2}\right]\left(4 a^{2}+\varpi^{2}\right)^{\frac{1}{2}}=4\right)$. This matching relation was first reported in the higher-order NLS model by Akhmediev's group [61,62], and in the NLS-MB system by Yang's group [33]. Figures 1(b) and 1(f) show the transformed nonbreathing modes in each 

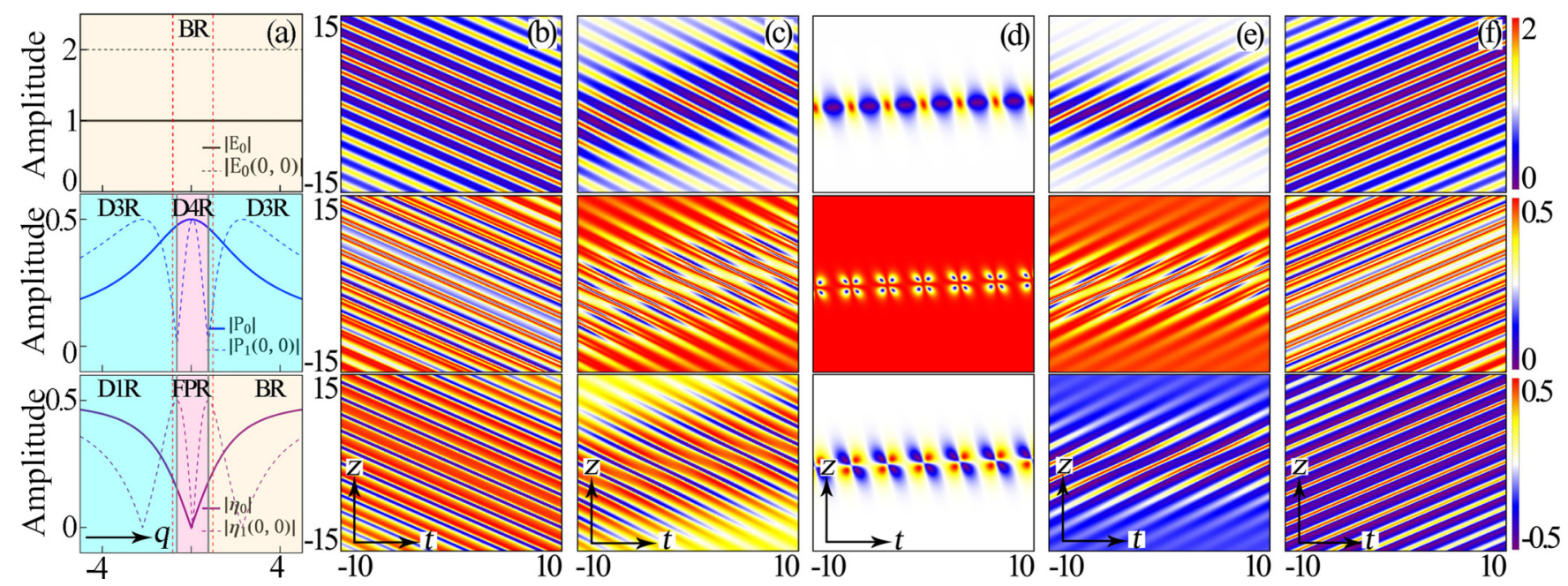

FIG. 1. Amplitude distributions of fundamental breathers (from top to bottom $\left|E_{1}\right|,\left|P_{1}\right|, \eta_{1}$ ) with $q=-0.83,-0.65,0,0.78,0.97$ (from left to right). Figure (a) shows the phase diagrams of fundamental breathers, namely, bright breathers in "BR" (bright regime); dark structures including dark three-valley and dark one-valley breathers in "D3R" (dark three-valley regime) and "D1R" (dark one-valley regime), respectively; four-petaled structures including dark four-valley and four-petaled breathers in "D4R" (dark four-valley regime) and "FPR" (four-petaled regime), respectively. Other parameters are $a=1, \omega=0, R=1.05, \alpha=\pi / 3$, and $\theta_{1}=\theta_{2}=0$.

component. These waves are the so-called "quasi-periodic modes", which are transformed from the "quasi-Akhmediev breathers." It has been demonstrated [63] that these nonbreathing modes can suffer perturbations although they are located on a nonzero background.

Based on the analysis above, the structure characteristics of fundamental breathers in the NLS-MB system are obtained completely. The whole picture is well depicted in Fig. 1(a). Note that this classification holds for the nonlinear superposition case of these fundamental breathers.

\section{SR BREATHER}

Nonlinear superposition of fundamental breathers can generate SR breathers in the NLS-MB system. The simplest case is the SR one-pair breather with the parameters $\alpha_{1}=-\alpha_{2}=$ $\alpha, R_{1}=R_{2}=R=1+\varepsilon$, where $\varepsilon$ is a small value $(\varepsilon \ll 1)$. The corresponding exact solution is obtained by the standard iteration of the Darboux transformation [see Appendix B]. In this case, the SR breather admits small localized perturbations on the plane wave at $z=0$ when $\theta_{1}+\theta_{2}=\pi$, and the perturbation amplitude is proportional to the value of $\varepsilon$.

Figure 2 shows the amplitude profiles of planewave backgrounds $\left(\left|E_{0}\right|,\left|P_{0}\right|, \eta_{0}\right)$ and the perturbations
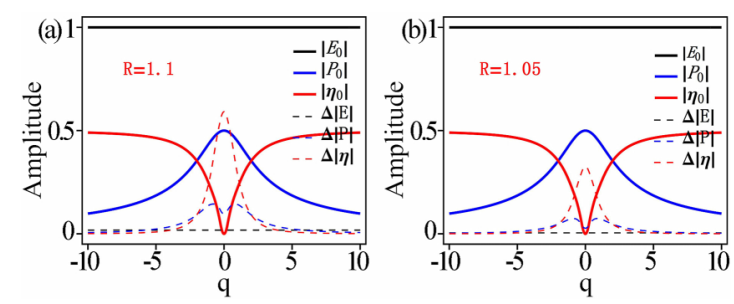

FIG. 2. Amplitude profiles of plane-wave backgrounds $\left(\left|E_{0}\right|,\left|P_{0}\right|, \eta_{0}\right)$ and the perturbations of SR breathers at $z=0$ ( $\Delta|E|, \Delta|P|, \Delta \eta$ ) versus $q$. (a) $R=1.1$; (b) $R=1.05$. Other parameters are $a=1, \omega=0, \alpha=\pi / 3$, and $\theta_{1}=\theta_{2}=\pi / 2$. at $z=0$ (i.e., $\quad \Delta|E|=|E|_{\max }-|E|_{\min }, \Delta|P|=|P|_{\max }-$ $\left.|P|_{\min }, \Delta \eta=\left|\eta_{\max }-\eta_{\min }\right|\right)$. As can be seen, the perturbation amplitudes decrease as $\varepsilon$ decreases, while the background amplitudes remain the same. In particular, the amplitudes $\left|E_{0}\right|$ and $\Delta|E|$ remains unchanged as $q$ varies, while the amplitudes for the matter wave components display different distributions due to the spatiotemporally balanced condition between them. Indeed, one can readily obtain the perturbation expression for the $E$ component, i.e,

\section{$\Delta E \approx-4 i a \varepsilon \cosh (i \alpha) \cos (2 a t \sin \alpha) \operatorname{sech}(2 a t \varepsilon \cos \alpha)$.}

This is the same as that obtained in the NLS systems [14]. Namely, the coupling effect arising from the matter wave components has no influence on the perturbation of the optical field. Thus, we shall choose a smaller value of $\varepsilon$ to make sure that the perturbation amplitudes can be small. However, one should note that for the special case $q=2 \omega,\left|P_{0}\right|$ and $\Delta \eta$ reach their maximums, while $\Delta|P|$ and $\eta_{0}$ will be small values. In this case, only the $P$ component admits a small dip perturbation (see the Sec. VI).

We then show the characteristics of SR breathers in Fig. 3. Three typical types of SR breathers are obtained via the analysis of the group and phase velocities.

First, when $V_{\mathrm{gr} 1} \neq V_{\mathrm{gr} 2}$ and $V_{\mathrm{grj}} \neq V_{\mathrm{phj}}$, the SR breathers formed by two quasi-Akhmediev breathers with different group velocities are obtained [Fig. 3(a)]. This is the generalization of the standard SR breathers in the NLS-MB system. However, note that the matter-wave SR modes can exhibit different structures. As shown in Fig. 3(a), two four-petaled SR breathers are observed in the $P$ and $\eta$ components, respectively.

Second, when $V_{\mathrm{grj}}=V_{\mathrm{phj}}, V_{\mathrm{gr} 3-\mathrm{j}} \neq V_{\mathrm{ph} 3-\mathrm{j}}$, we observe the interesting half-transition states of SR breathers [Fig. 3(b)]. Namely, a breather is converted to a nonbreathing wave when $V_{\mathrm{grj}}=V_{\mathrm{phj}}$, while the other remains the nature of the breather when $V_{\text {gr3-j }} \neq V_{\text {ph3-j. }}$. In contrast to the standard SR wave, the 


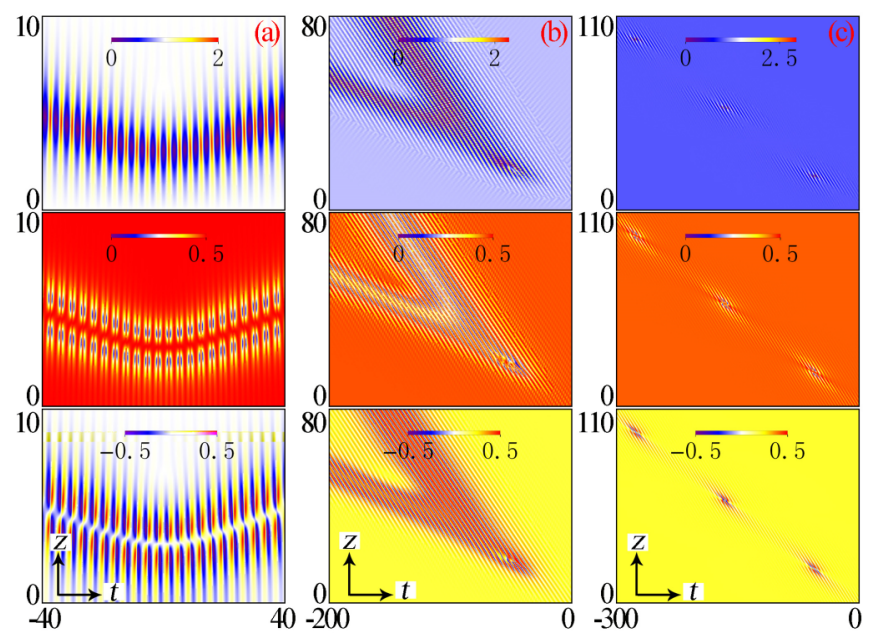

FIG. 3. Amplitude distributions of SR breather structures in the $E, P, \eta$ components versus $q(q=0,-0.834,-0.906$ from left to right), (a) the standard SR breathers, (b) half-transition states, (c) SR bound states. Other parameters are $a=1, \omega=0, R=1.05, \alpha=$ $\pi / 3$, and $\theta_{1}=\theta_{2}=\pi / 2$.

small-amplitude perturbation is amplified a little slowly and subsequently becomes a mix of breathing and nonbreathing waves.

Third, when $V_{\mathrm{gr} 1}=V_{\mathrm{gr} 2}$, an SR bound state is observed in Fig. 3(c). In this particular case, the small-amplitude perturbation forms a bound state on the plane-wave background with a long-period oscillation. This oscillation stems from the nonvanishing difference of phase velocity. The smaller that the difference of phase velocity is, the longer that the oscillation period will have (not shown). If the difference of phase velocity is vanishing, the oscillation is gone, which is the so-called full-suppression SR state reported before $[29,30,55]$. We will show below that this bound state corresponds exactly to the vanishing MI growth rate.

\section{EXACT LINK BETWEEN SR BREATHERS AND MI}

Establishing an exact link between SR breathers and MI is one crucial step in both the SR breather theory and the analytic MI description. It has been demonstrated in the scalar NLSE hierarchy that the absolute difference of group velocities of scalar SR breathers coincides exactly with the linear MI growth rate [26]. Here we will find out whether this exact link is valid for the complex coupled case. For the coupled NLS-MB system, SR breathers in each component share the same group velocity although their structures can be different. Thus, the absolute difference of group velocities of SR breathers can be given by the following simple expression:

$$
\Delta V_{\mathrm{gr}}=\left|V_{\mathrm{gr} 1}-V_{\mathrm{gr} 2}\right| .
$$

The explicit expression of $\Delta V_{\mathrm{gr}}$ for the each component of the NLS-MB system is written as

$$
\begin{aligned}
\Delta V_{\mathrm{gr}} & =\mid \varrho-\frac{\rho \eta_{i}}{\eta_{r}}+\frac{2 k}{\eta_{r}}\left[\frac{\rho \eta_{i}+\eta_{r}(\varrho+\varpi)}{\rho^{2}+(\varrho+\varpi)^{2}}\right] \\
& +\frac{2 k}{\eta_{r}}\left[\frac{\rho \eta_{i}+\eta_{r}(\varrho-\varpi)}{\rho^{2}+(\varrho-\varpi)^{2}}\right] \mid,
\end{aligned}
$$

where $\rho=-a(R+1 / R) \cos \alpha, \varrho=a(R-1 / R) \sin \alpha, \eta_{r}=$ $a(R-1 / R) \cos \alpha$, and $\eta_{i}=a(R+1 / R) \sin \alpha$. Considering the condition of SR waves, $R=1+\varepsilon, \varepsilon \ll 1$ (which indicates $\varepsilon^{2} \rightarrow 0$ ), we shall omit the terms with respect to $\varepsilon^{2}$. Thus, Eq. (14) is rewritten as a simpler form:

$$
\Delta V_{\mathrm{gr}}=\left|\frac{2 a^{2} \sin 2 \alpha}{\eta_{r}}\left(1-\frac{4 k}{4 a^{2} \cos ^{2} \alpha+\varpi^{2}}\right)\right| .
$$

Obviously, when $\Delta V_{\mathrm{gr}}=0$, which indicates $4 a^{2} \cos ^{2} \alpha+$ $\varpi^{2}=4 k$, the SR bound state is obtained. This bound state shows a long-period oscillation phenomenon excited from small-amplitude perturbation, shown in Fig. 3(c).

Let us then turn our attention to the linear MI analysis, in which the MI criterion and growth rate of smallamplitude perturbations on a plane-wave background can be studied precisely. Perturbed backgrounds $E_{0 p}, P_{0 p}$, and $\eta_{0 p}$ are obtained via adding small amplitude perturbed Fourier modes $m_{1}, m_{2}$, and $m_{3}$, i.e., $E_{0 p}=\left(a+m_{1}\right) e^{i \theta}, P_{0 p}=$ $i k\left(a+m_{2}\right) e^{i \theta}$, and $\eta_{0 p}=k\left(\omega-q / 2+m_{3}\right)$, where $m_{1}=$ $u_{1} \cos (K z-\Omega t)+i v_{1} \sin (K z-\Omega t), m_{2}=u_{2} \cos (K z-$ $\Omega t)+i v_{2} \sin (K z-\Omega t)$, and $m_{3}=u_{3} \cos (K z-\Omega t)$. Here $u_{1}, u_{2}, u_{3}, v_{1}$, and $v_{2}$ are small numbers describing the amplitudes of perturbations, $\Omega$ represents perturbed frequency, and the wave number $K$ is assumed to be complex. Followed by the standard linearization process, a substitution of $E_{0 p}, P_{0 p}$, and $\eta_{0 p}$ into Eq. (7) yields the dispersion relation between $K$ and $\Omega$. The imaginary part of $K$ leads to MI. The latter is described by the MI growth rate, which is defined by $G=|\operatorname{Im}\{K\}|$. The corresponding explicit expression is given by

$$
G=\frac{1}{2} \mid \Omega \sqrt{\Omega^{2}-4 a^{2}}\left(1-\frac{4 k}{4 a^{2}-\Omega^{2}+\varpi^{2}}\right),
$$

where $|\Omega|<2 a$. Obviously, the initial perturbed frequency of SR breathers $\Omega=2 a \sin \alpha$ falls within this MI region $|\Omega|<$ $2 a$. Substituting $\Omega=2 a \sin \alpha$ into Eq. (16), one can readily obtain the linear MI growth rate for the initial state of SR breathers as follows:

$$
G_{\mathrm{sr}}=\left|a^{2} \sin 2 \alpha\left(1-\frac{4 k}{4 a^{2} \cos ^{2} \alpha+\varpi^{2}}\right)\right| .
$$

Remarkably, a simple comparison between $G_{\mathrm{sr}}$ and $\Delta V_{\mathrm{gr}}$ shows an exact link

$$
G_{\mathrm{sr}}=\Delta V_{\mathrm{gr}} \eta_{r} / 2
$$

where $\eta_{r}=a(R-1 / R) \cos \alpha$. Thus, despite the complexity caused by resonant interaction, an excellent consistency between SR breathers and linear MI growth rate is still obtained. It shows that the absolute difference of group velocities of SR breathers coincides exactly with the linear MI growth rate. This link is consistent with that in the NLS models [26]. Clearly, for fixed $R$ and $\alpha$, one obtains $G_{\mathrm{sr}} \rightarrow 0$ as $\Delta V_{\mathrm{gr}} \rightarrow 0$. Eventually, the SR bound state $\Delta V_{\mathrm{gr}}=0$ corresponds to the vanishing MI growth rate $G_{\mathrm{sr}}=0$. This exact link reveals the universal rule of SR waves in many nonlinearly driven systems. 



FIG. 4. Maximum and minimum amplitudes of SR breather in the $E$ (a) and $P$ (b) components. Parameters are $a=1, \omega=0, \alpha=$ $\pi / 3$, and $\theta_{1}=\theta_{2}=\pi / 2$.

\section{MI DESCRIBED BY DARK SR BREATHER}

Up to now, MI dynamics described by the SR breathers is confined to the bright breather structure. However, as shown above, for the coupled systems, SR breathers can exhibit a dark structure. This unique property is absent in the scalar systems. In the following, we will show clearly the MI nature of the dark SR breather.

Figure 4 shows the amplitude evolution of SR breathers in each component. The amplitude amplification and attenuation are tracked by the maximum and minimum as $z$ increases. For the optical wave component $E$, the small perturbation is amplified and attenuated exponentially at the first four propagation distances. This is the initial MI stage, which coincides exactly with the results of linear stability analysis obtained above. After that, the wave maximum and minimum are oscillating near the thresholds $|E|_{\max }=2$ and $|E|_{\min }=0$. This is referred to as the nonlinear stage of MI, which exhibits two quasi-Akhmediev breathers propagation along different directions.

For the matter wave component $P$, however, an MI evolution is captured. In sharp contrast to the component $E$, we find that a small dip perturbation of dark SR breather suffers a completely exponential decay in the component $P$. After the initial exponential attenuation, this nonlinear MI stage shows two quasi-Akhmediev breathers with dark structures. It should be pointed out that a small dip perturbation on a planewave background associated with the continuous spectrum has been used to excite MI in the standard NLS system [64]. However, the process is different from that reported here. This is because the MI evolution in Ref. [64] is essentially a process involving both the amplitude amplification and attenuation. The intriguing attenuation process reported here helps us to understand the exact descriptions of the complete MI process in complex coupled systems.

\section{DISCUSSION}

To connect the analytical results reported in this work with more actual physical context, let us discuss briefly the applicability and possible observation of the analytic prediction.

First, we will analyze the applicability of the analytical results. As shown above, the NLS-MB system possesses both MI and modulation stability regions in the range of perturbed frequency. SR breathers in each region can exist but exhibit different nonlinear evolution. Thus, our results provide flexibility for the excitations of different SR breathers. Owing to the exact link Eq. (18), one simple way to observe SR breathers is to identify the MI criterion and growth rate by the linear stability analysis. Namely, one can estimate the absolute difference of group velocities of SR breathers by the linear growth rate.

After the preliminary linear analysis above, the next step is to prepare the corresponding initial excitations. Of course, the exact initial conditions can be extracted from the exact solutions at $z=0$. However, there are approximate initial forms to excite SR breathers more simply. The initial modulation for the light field is given by Eq. (18). Moreover, as suggested in Refs. [26,28], a more general form can be used to excite SR breathers as long as the width of initial state can be comparable with $b \approx 1 /(2 a \varepsilon \cos \alpha)$. Importantly, this initial condition has been realized in a fiber by the initial intensity and phase modulations [16]. For the matter wave components, as shown in Ref. [41], the pure plane waves (viz, $P_{0}, \eta_{0}$ ) can be chosen as the initial conditions without affecting the linear stage of MI.

After the applicability analysis above, we then discuss the possible observation of these SR breather structures. Our analysis starts from Eq. (1) with the documented material parameters suggested in Refs. [57] and [16], where SIT solitons in an erbium-doped fiber waveguide and SR breathers in standard NLS fiber system have been observed, respectively. At the first step, the cooling of the erbiumdoped fiber to the cryogenic temperature of $4.2 \mathrm{~K}$ is a prerequisite to increase the dipole decay time to nanoseconds. Then, an effective though truncated plane-wave background should be prepared by the modern pulse shaping, which may give rise to SR breather dynamics reported above. Indeed, this initial background condition can be achieved by the dispersion engineering and management [65] as well as the modification of the transition electric dipole moment of doping ions [50]. For example, we may consider the case that the operation wavelength of each mode is nearly $1.55 \mu \mathrm{m}$ with the dispersion coefficient $\beta_{2}=2.76 \times 10^{7} \mathrm{ps}^{2} / \mathrm{km}$ in the anomalous regime, and the Kerr nonlinearity parameter $\gamma=12.8 \mathrm{~W}^{-1} / \mathrm{km}$. Parameters $\delta=0, \hbar=6.626 /(2 \pi) \times$ $10^{-34} \mathrm{~J} \mathrm{~s}, c=3 \times 10^{8} \mathrm{~m} / \mathrm{s}, \mathrm{n}=1.454$ are used from the pioneering experiment [57]. By using the transformations Eq. (2) with the conditions Eqs. (4) and (6), the correspondence between theory and experiment can be retrieved. Here we assume that the initial pulse width is chosen as $t_{s}=500 \mathrm{ps}$ with the peak power $p_{n}=p_{s}=8.6 \mathrm{~W}$. Under these assumptions, optical SR breathers, predicted in Fig. 4(a) should be triggered by modulation instability with the peak power around $11.7 \mathrm{~W}$. However, one should note that the observation of the matter-wave counterpart remains particularly challenging, as mentioned in Refs. [41,57].

\section{CONCLUSION}

In summary, we have investigated SR breathers formed by the resonant interaction in an erbium-doped fiber. We have demonstrated explicitly that the optical wave component always features a bright structure that describes the MI development from localized small perturbations, while the SR matter wave breathers exhibit more complicated dynamical evolution. SR breathers in each component involve the half-transition and bound states arising from the resonant 
interaction. Despite the complexity, we have established the exact link between SR breathers and MI by showing that the absolute difference of group velocities coincides exactly with the linear MI growth rate. Further, we have shown how a small dip perturbation of dark SR breathers in the matter wave component induces an intriguing MI process of completely exponential decay of perturbation. These results will enrich our understanding on the MI development in complex coupled light-matter interaction systems.

\section{ACKNOWLEDGMENTS}

This work has been supported by the National Natural Science Foundation of China (NSFC) (Grants No. 11705145, No. 11875220, No. 11434013, and No. 11425522), Natural Science Basic Research Plan in Shaanxi Province of China (Grant No. 2018JQ1003), and Major Basic Research Program of Shaanxi Province Natural Science Foundation Research Project (Grants No. 2017KCT-12 and No. 2017ZDJC-32).

\section{APPENDIX A: EXACT SOLUTIONS OF FUNDAMENTAL BREATHERS}

The fundamental breather solutions of the coupled NLS-MB Eq. (7) on the plane-wave Eq. (8) are given by

$$
\begin{gathered}
E_{1}=e^{i \theta}\left\{a+\frac{2 \rho \varphi_{12}}{\varphi_{11}+\varphi_{22}}\right\} \\
P_{1}=i k e^{i \theta}\left\{\frac{-\left(\wp_{1} \varphi_{11}-\wp_{2} \varphi_{22}\right)\left[2 i \rho(q-2 \omega) \varphi_{12}+a\left(\wp_{1} \varphi_{11}-\wp_{2} \varphi_{22}\right)\right]-4 a \rho^{2} \varphi_{12}^{2}}{\left(\varphi_{11}+\varphi_{22}\right)^{2}\left(\rho^{2}+s_{2}^{2}\right)}\right\}, \\
\eta_{1}=-k\left\{\frac{q-2 \omega}{2}+\frac{2\left[a \rho\left(s_{2} \varpi_{1}-i \rho \varpi_{2}\right)-2 \varphi_{11} \varphi_{22} \rho^{2}(q-2 \omega)\right]}{\left(\varphi_{11}+\varphi_{22}\right)^{2}\left(\rho^{2}+s_{2}^{2}\right)}\right\},
\end{gathered}
$$

where

$$
\begin{aligned}
\varpi_{j} & =\left(\varphi_{12} \pm \varphi_{21}\right)\left(\varphi_{11} \pm \varphi_{22}\right), \quad \wp=\left(\rho-i s_{2}\right) \varphi_{11}-\left(\rho+i s_{2}\right) \varphi_{22}, \\
\varphi_{j j} & = \pm \psi_{1} \sinh \sigma_{1}+\psi_{2} \cosh \sigma_{1}-\cos \left(\sigma_{2} \mp \alpha\right), \quad \sigma_{1}=\eta_{r 1}\left(t-V_{\mathrm{gr} 1} z\right)+\mu_{1}, \\
\varphi_{j 3-j} & =\cosh \left(\sigma_{1} \mp i \alpha\right) \mp i \psi_{1} \sin \sigma_{2}-\psi_{2} \cos \sigma_{2}, \quad \sigma_{2}=\eta_{i 1}\left(t-V_{\mathrm{ph} 1} z\right)-\theta_{1}, \\
V_{\mathrm{gr} 1} & =v_{1}+v_{2} \frac{\eta_{i 1}}{\eta_{r 1}}, \quad V_{\mathrm{ph} 1}=v_{1}-v_{2} \frac{\eta_{r 1}}{\eta_{i 1}}, \quad v_{1}=-\frac{2 k s_{1}}{\rho^{2}+s_{1}^{2}}-\frac{\varrho}{2}+q, \quad v_{2}=-\frac{2 k \rho}{\rho^{2}+s_{1}^{2}}+\frac{\rho}{2},
\end{aligned}
$$

with $\wp_{j}=\rho \mp i s_{2}, s_{1}=\varrho-q+2 \omega, s_{2}=\varrho+q-2 \omega, \rho=-a(R+1 / R) \cos \alpha, \varrho=a(R-1 / R) \sin \alpha, \eta_{r 1}=a(R-1 / R)$ $\cos \alpha, \eta_{i 1}=a(R+1 / R) \sin \alpha, \psi_{1}=(1 / R-R) / 2$, and $j=1,2$.

\section{APPENDIX B: EXACT SOLUTIONS OF SR BREATHERS}

The SR breather solutions of the NLS-MB Eq. (7) on the plane-wave Eq. (8) are presented as follows:

$$
\begin{gathered}
E_{2}=e^{i \theta}\left\{a+\frac{2 \rho \varrho\left[(\varrho+i \rho)\left(\varphi_{11} \phi_{12}+\varphi_{12} \phi_{22}\right)+(\varrho-i \rho)\left(\varphi_{12} \phi_{11}+\varphi_{22} \phi_{12}\right)\right]}{\left[\rho^{2}\left(\varphi_{11} \phi_{22}-\varphi_{21} \phi_{12}-\varphi_{12} \phi_{21}+\varphi_{22} \phi_{11}\right)+\varrho^{2}\left(\varphi_{11}+\varphi_{22}\right)\left(\phi_{11}+\phi_{22}\right)\right]}\right\}, \\
P_{2}=i k e^{i \theta}\left\{\frac{2 \Delta_{3} \Xi_{1} \Xi_{2}+\Delta_{1} \Xi_{2}^{2}+\Delta_{2} \Xi_{1}^{2}}{\left(\rho^{2} \varphi_{12} \varphi_{21}+\delta_{1} \delta_{2}\right)\left(\Xi_{1} \Xi_{4}-\Xi_{2} \Xi_{3}\right)}\right\}, \\
\eta_{2}=i k\left\{\frac{\Delta_{3}\left(\Xi_{1} \Xi_{4}+\Xi_{2} \Xi_{3}\right)+\Delta_{1} \Xi_{2} \Xi_{4}+\Delta_{2} \Xi_{1} \Xi_{3}}{\left(\rho^{2} \varphi_{12} \varphi_{21}+\delta_{1} \delta_{2}\right)\left(\Xi_{1} \Xi_{4}-\Xi_{2} \Xi_{3}\right)}\right\},
\end{gathered}
$$

where

$$
\begin{aligned}
\Delta_{1}= & 2 \rho(\omega-q / 2) \varphi_{21} \delta_{1}+a\left(\delta_{1}^{2}-\rho^{2} \varphi_{21}^{2}\right), \\
\Delta_{2}= & 2 \rho(\omega-q / 2) \varphi_{12} \delta_{2}+a\left(\delta_{2}^{2}-\rho^{2} \varphi_{12}^{2}\right), \\
\Delta_{3}= & i \rho a\left(\varphi_{12} \delta_{1}+\varphi_{21} \delta_{2}\right)-i(\omega-q / 2)\left(\delta_{1} \delta_{2}-\rho^{2} \varphi_{12} \varphi_{21}\right), \\
\delta_{j}= & \left(s_{1} \mp i \rho\right) \varphi_{11} / 2+\left(s_{1} \pm i \rho\right) \varphi_{22} / 2, \Xi_{1,4}=\left(\chi_{1} s_{2} \mp i \chi_{2} \rho\right) / 2, \\
\Xi_{2}= & -i \rho\left[\varrho^{2} \phi_{12}\left(\varphi_{11}+\varphi_{12}\right)^{2}+i \rho \varrho\left(\varphi_{11}+\varphi_{12}\right)\left(\varphi_{11} \phi_{12}-\varphi_{12} \phi_{11}+\varphi_{12} \phi_{22}-\varphi_{22} \phi_{12}\right)\right. \\
& \left.-\rho^{2}\left(-\varphi_{12}^{2} \phi_{21}+\varphi_{11} \varphi_{12} \phi_{22}-\varphi_{11} \varphi_{22} \phi_{12}+\varphi_{12} \varphi_{22} \phi_{11}\right)\right], \\
\Xi_{3}= & -i \rho\left[\varrho^{2} \phi_{21}\left(\varphi_{11}+\varphi_{12}\right)^{2}-i \rho \varrho\left(\varphi_{11}+\varphi_{12}\right)\left(\varphi_{11} \phi_{21}-\varphi_{21} \phi_{11}+\varphi_{21} \phi_{22}-\varphi_{22} \phi_{21}\right)\right. \\
& \left.-\rho^{2}\left(-\varphi_{21}^{2} \phi_{12}+\varphi_{11} \varphi_{21} \phi_{22}-\varphi_{11} \varphi_{22} \phi_{21}+\varphi_{21} \varphi_{22} \phi_{11}\right)\right], \\
\chi_{1}= & \left(\varphi_{11}+\varphi_{22}\right)\left[\varrho^{2}\left(\phi_{11}+\phi_{22}\right)\left(\varphi_{11}+\varphi_{22}\right)+\rho^{2}\left(\varphi_{11} \phi_{22}+\varphi_{22} \phi_{11}-\varphi_{12} \phi_{21}-\varphi_{21} \phi_{12}\right)\right],
\end{aligned}
$$




$$
\begin{aligned}
\chi_{2}= & 2 i \rho \varrho\left(\varphi_{11}+\varphi_{22}\right)\left(\varphi_{12} \phi_{21}-\varphi_{21} \phi_{12}\right)+\varrho^{2}\left(\phi_{11}-\phi_{22}\right)\left(\varphi_{11}+\varphi_{22}\right)^{2} \\
& -\rho^{2}\left(\varphi_{11}-\varphi_{22}\right)\left(\varphi_{11} \phi_{22}+\varphi_{22} \phi_{11}-\varphi_{12} \phi_{21}-\varphi_{21} \phi_{12}\right),
\end{aligned}
$$

where

$$
\begin{aligned}
& \phi_{j j}= \pm \psi_{1} \sinh \varsigma_{1}+\psi_{2} \cosh \varsigma_{1}-\cos \left(\varsigma_{2} \pm \alpha\right), \phi_{j 3-j}=\cosh \left(\varsigma_{1} \pm i \alpha\right) \mp i \psi_{1} \sin \varsigma_{2}-\psi_{2} \cos \varsigma_{2}, \\
& \varsigma_{1}=\eta_{r 2}\left(t-V_{\mathrm{gr} 2} z\right)+\mu_{2}, \varsigma_{2}=\eta_{i 2}\left(t-V_{\mathrm{ph} 2} z\right)-\theta_{2}, V_{\mathrm{gr} 2}=v_{1}+v_{2} \frac{\eta_{i 2}}{\eta_{r 2}}, V_{\mathrm{ph} 2}=v_{1}-v_{2} \frac{\eta_{r 2}}{\eta_{i 2}}, \\
& \nu_{1}=\frac{2 k s_{2}}{\rho^{2}+s_{2}^{2}}+\frac{\varrho}{2}+q, \nu_{2}=\frac{2 k \rho}{\rho^{2}+s_{2}^{2}}+\frac{\rho}{2},
\end{aligned}
$$

with $\psi_{2}=(1 / R+R) / 2, \eta_{r 2}=a(R-1 / R) \cos \alpha$, and $\eta_{i 2}=-a(R+1 / R) \sin \alpha$. Other parameters are in Appendix A.

[1] V. E. Zakharov and L. A. Ostrovsky, Physica D: Nonlin. Phenom. 238, 540 (2009).

[2] J. M. Dudley, F. Dias, M. Erkintalo, and G. Genty, Nat. Photon. 8, 755 (2014).

[3] B. Kibler, J. Fatome, C. Finot, G. Millot, F. Dias, G. Genty, N. Akhmediev, and J. M. Dudley, Nat. Phys. 6, 790 (2010).

[4] S. Toenger, T. Godin, C. Billet, F. Dias, M. Erkintalo, G. Genty, and J. M. Dudley, Sci. Rep. 5, 10380 (2015).

[5] Y. V. Bludov, V. V. Konotop, and N. Akhmediev, Phys. Rev. A 80, 033610 (2009).

[6] W. M. Moslem, P. K. Shukla, and B. Eliasson, Europhys. Lett. 96, 25002 (2011).

[7] A. N. Ganshin, V. B. Efimov, G. V. Kolmakov, L. P. MezhovDeglin, and P. V. E. McClintock, Phys. Rev. Lett. 101, 065303 (2008).

[8] S. Trillo and A. Valiani, Opt. Lett. 35, 3967 (2010).

[9] B. Wetzel, M. Erkintalo, G. Genty, K. Hammani, B. Kibler, J. Fatome, C. Finot, F. Dias, N. Akhmediev, G. Millot, and J. M. Dudley, Optoelectron Commun SPIE Newsroom 10, 1201104 (2011).

[10] N. Akhmediev and A. Ankiewicz, Solitons: Nolinear Pulses and Beams (Chapman and Hall, London, 1997).

[11] J. M. Dudley, G. Genty, F. Dias, B. Kibler, and N. Akhmediev, Opt. Express 17, 21497 (2009).

[12] B. Kibler, J. Fatome, C. Finot, G. Millot, G. Genty, B. Wetzel, N. Akhmediev, F. Dias, and J. M. Dudley, Sci. Rep. 2, 463 (2012).

[13] N. Akhmediev, V. I. Korneev, Theor. Math. Phys. 69, 1089 (1986); N. Akhmediev, Nature (London) 413, 267 (2001).

[14] V. E. Zakharov and A. A. Gelash, Phys. Rev. Lett. 111, 054101 (2013).

[15] A. A. Gelash and V. E. Zakharov, Nonlinearity 27, R1 (2014).

[16] B. Kibler, A. Chabchoub, A. Gelash, N. Akhmediev, and V. E. Zakharov, Phys. Rev. X 5, 041026 (2015).

[17] S. Flach and A. V. Gorbach, Phys. Rep. 467, 1 (2008).

[18] F. Lederer, G. I. Stegeman, D. N. Christodoulides, G. Assanto, M. Segev, and Y. Silberberg, Phys. Rep. 463, 1 (2008).

[19] D. H. Peregrine, J. Aust. Math. Soc. Series B 25, 16 (1983).

[20] M. Erkintalo, K. Hammani, B. Kibler, C. Finot, N. Akhmediev, J. M. Dudley, and G. Genty, Phys. Rev. Lett. 107, 253901 (2011).

[21] O. Kimmoun, H. C. Hsu, H. Branger, M. S. Li, Y. Y. Chen, C. Kharif, M. Onorato, E. J. R. Kelleher, B. Kibler, N. Akhmediev, and A. Chabchoub, Sci. Rep. 6, 28516 (2016).
[22] O. Kimmoun, H. C. Hsu, B. Kibler, and A. Chabchoub, Phys. Rev. E 96, 022219 (2017).

[23] G. El, A. V. Gurevich, V. V. Khodorovskii, and A. L. Krylov, Phys. Lett. A 177, 357 (1993); G. Biondini and D. Mantzavinos, Phys. Rev. Lett. 116, 043902 (2016); Commun. Pure Appl. Math. 70, 2300 (2017).

[24] F. Baronio, M. Conforti, A. Degasperis, S. Lombardo, M. Onorato, and S. Wabnitz, Phys. Rev. Lett. 113, 034101 (2014); F. Baronio, S. Chen, P. Grelu, S. Wabnitz, and M. Conforti, Phys. Rev. A 91, 033804 (2015); L. C. Zhao and L. M. Ling, J. Opt. Soc. Am. B 33, 850 (2016); L. C. Zhao, L. M. Ling, and Z. Y. Yang, Phys. Rev. E 97, 022218 (2018).

[25] N. Akhmediev, A. Ankiewicz, and M. Taki, Phys. Lett. A 373, 675 (2009); N. Akhmediev, J. M. Soto-Crespo, and A. Ankiewicz, ibid. 373, 2137 (2009); Phys. Rev. A 80, 043818 (2009).

[26] C. Liu, Z. Y. Yang, and W. L. Yang, Chaos 28, 083110 (2018).

[27] B. Kibler, A. Chabchoub, A. Gelash, N. Akhmediev, and V. E. Zakharov, in Nonlinear Guided Wave Optics: A Testbed for Extreme Waves, edited by S. Wabnitz (IOP Publishing, Bristol, UK, 2017), Chap. 7.

[28] A. A. Gelash, Phys. Rev. E 97, 022208 (2018).

[29] C. Liu, L. Wang, Z. Y. Yang, and W. L. Yang, arXiv:1708.03781 (2017).

[30] C. Liu, Y. Ren, Z. Y. Yang, and W. L. Yang, Chaos 27, 083120 (2017).

[31] J. S. He, L. J. Guo, Y. S. Zhang, and A. Chabchoub, Proc. R. Soc. London A 470, 20140318 (2014).

[32] S. Chen and L. Y. Song, Phys. Rev. E 87, 032910 (2013); S. Chen, P. Grelu, and J. M. Soto-Crespo, ibid. 89, 011201 (2014); S. Chen, J. M. Soto-Crespo, and P. Grelu, ibid. 90, 033203 (2014); W. R. Sun, D. Y. Liu, and X. Y. Xie, Chaos 27, 043114 (2017).

[33] Y. Ren, Z. Y. Yang, C. Liu, and W. L. Yang, Phys. Lett. A 379, 2991 (2015); Y. Ren, Z. Y. Yang, C. Liu, W. H. Xu, and W. L. Yang, Eur. Phys. J. D 70, 187 (2016).

[34] L. C. Zhao and J. Liu, J. Opt. Soc. Am. B 29, 3119 (2012).

[35] A. Degasperis and S. Lombardo, Phys. Rev. E 88, 052914 (2013).

[36] C. Liu, Z. Y. Yang, L. C. Zhao, and W. L. Yang, Phys. Rev. A 89, 055803 (2014).

[37] C. Liu, Z. Y. Yang, L. C. Zhao, and W. L. Yang, Ann. Phys. 362, 130 (2015). 
[38] F. Baronio, A. Degasperis, M. Conforti, and S. Wabnitz, Phys. Rev. Lett. 109, 044102 (2012).

[39] L. C. Zhao and J. Liu, Phys. Rev. E 87, 013201 (2013).

[40] F. Baronio, M. Conforti, A. Degasperis, and S. Lombardo, Phys. Rev. Lett. 111, 114101 (2013).

[41] S. Chen, Y. Ye, F. Baronio, Y. Liu, X. M. Cai, and P. Grelu, Opt. Express 25, 29687 (2017).

[42] B. L. Guo and L. M. Ling, Chin. Phys. Lett. 28, 110202 (2011).

[43] D. L. Mills and E. Burstein, Rep. Prog. Phys. 37, 817 (1974).

[44] D. V. Skryabin, D. N. Krizhanovskii, M. S. Skolnick, E. A. Cerda-Méndez, and R. Hartley, Nat. Photonics 6, 204 (2012).

[45] H. A. Haus, Rev. Mod. Phys. 51, 331 (1979).

[46] G. H. M. van Tartwijk and G. P. Agrawal, J. Opt. Soc. Am. B 14, 2618 (1997).

[47] R. W. Ziolkowski, J. M. Arnold, and D. M. Gogny, Phys. Rev. A 52, 3082 (1995).

[48] Q.-H. Park and R. W. Boyd, Phys. Rev. Lett. 86, 2774 (2001).

[49] S. L. McCall and E. L. Hahn, Phys. Rev. Lett. 18, 908 (1967).

[50] L. Allen and J. H. Eberly, Optical Resonance and Two-Level Atoms (Wiley, New York, 1975).

[51] A. Kundu, Theor. Math. Phys. 167, 800 (2011).

[52] J. He, S. W. Xu, and K. Porsezian, Phys. Rev. E 86, 066603 (2012).

[53] S. Xu, K. Porsezian, J. He, and Y. Cheng, Phys. Rev. E 88, 062925 (2013); L. Wang, X. Wu, and H. Y. Zhang, Phys. Lett. A 382, 2650 (2018).
[54] S. Xu, J. He, and K. Porsezian, Wave Motion 80, 82 (2018).

[55] Y. Ren, X. Wang, C. Liu, Z. Y. Yang, and W. L. Yang, Commun. Nonlinear Sci. Numer. Simul. 63, 161 (2018).

[56] M. Nakazawa, E. Yamada, and H. Kubota, Phys. Rev. A 44, 5973 (1991).

[57] M. Nakazawa, Y. Kimura, K. Kurokawa, and K. Suzuki, Phys. Rev. A 45, R23 (1992).

[58] A. I. Maimistov and E. A. Manykin, Sov. Phys. JETP. 85, 1177 (1983).

[59] K. Binmore and J. Davies, Calculus: Concepts and Methods (Cambridge University Press, Cambridge, 2002).

[60] L. Ling, L. C. Zhao, and B. Guo, Commun. Nonlinear Sci. Numer. Simul. 32, 285 (2016).

[61] A. Chowdury, A. Ankiewicz, and N. Akhmediev, Proc. R. Soc. London A 471, 20150130 (2015).

[62] A. Chowdury, D. J. Kedziora, A. Ankiewicz, and N. Akhmediev, Phys. Rev. E 91, 032928 (2015).

[63] C. Liu, Z. Y. Yang, L. C. Zhao, L. Duan, G. Yang, and W. L. Yang, Phys. Rev. E 94, 042221 (2016); C. Liu, Z. Y. Yang, L. C. Zhao, and W. L. Yang, ibid. 91, 022904 (2015).

[64] A. E. Kraych, P. Suret, G. El, and S. Randoux, arXiv:1805.05074.

[65] A. Hasegawa, Y. Kodama, and A. Maruta, Opt. Fiber Technol. 3, 197 (1997). 